\title{
BMJ Open Research protocol for the Picture Talk Project: a qualitative study on research and consent with remote Australian Aboriginal communities
}

\author{
Emily F M Fitzpatrick, ${ }^{1,2}$ Maureen Carter, ${ }^{3}$ June Oscar, ${ }^{4,5}$ Tom Lawford, $^{6}$ \\ Alexandra L C Martiniuk, ${ }^{7,8,9}$ Heather A D'Antoine, ${ }^{10}$ Elizabeth J Elliott ${ }^{1,2}$
}

To cite: Fitzpatrick EFM, Carter M, Oscar J, et al. Research protocol for the Picture Talk Project: a qualitative study on research and consent with remote Australian Aboriginal communities. BMJ Open 2017;7:e018452. doi:10.1136/ bmjopen-2017-018452

- Prepublication history and additional material for this paper are available online. To view these files, please visit the journal online (http://dx.doi. org/10.1136/bmjopen-2017018452).

Received 18 July 2017 Revised 19 September 2017 Accepted 23 0ctober 2017

CrossMark

For numbered affiliations see end of article.

Correspondence to Dr Emily F M Fitzpatrick; emilyfitzp@gmail.com

\section{ABSTRACT}

Introduction Research with Indigenous populations is not always designed with cultural sensitivity. Few publications evaluate or describe in detail seeking consent for research with Indigenous participants. When potential participants are not engaged in a culturally respectful manner, participation rates and research quality can be adversely affected. It is unethical to proceed with research without truly informed consent.

Methods and analysis We describe a culturally appropriate research protocol that is invited by Aboriginal communities of the Fitzroy Valley in Western Australia. The Picture Talk Project is a research partnership with local Aboriginal leaders who are also chief investigators. We will interview Aboriginal leaders about research, community engagement and the consent process and hold focus groups with Aboriginal community members about individual consent. Cultural protocols will be applied to recruit and conduct research with participants. Transcripts will be analysed using NViv010 qualitative software and themes synthesised to highlight the key issues raised by the community about the research process. This protocol will guide future research with the Aboriginal communities of the Fitzroy Valley and may inform the approach to research with other Indigenous communities of Australia or the world. It must be noted that no community is the same and all research requires local consultation and input. To conduct culturally sensitive research, respected local people from the community who have knowledge of cultural protocol and language are engaged to guide each step of the research process from the project design to the delivery of results.

Ethics and dissemination Ethics approval was granted by the University of Sydney Human Research Ethics Committee (No. 2012/348, reference:14760), the Western Australia Country Health Service Ethics Committee (No. 2012:15), the Western Australian Aboriginal Health Ethics Committee and reviewed by the Kimberley Aboriginal Health Planning Forum Research Sub-Committee (No. 2012-008). Results will be disseminated through peer review articles, a local Fitzroy Valley report and conference presentations.

\section{INTRODUCTION}

When seeking consent for research with Indigenous people, time is rarely taken to reflect on the process. ${ }^{12}$ If research is not

\section{Strengths and limitations of this study}

- This is one of few studies reporting Aboriginal community understanding of research. It is led by a research team that includes Aboriginal leaders, experienced public health researchers and clinicians and is conducted in a way that is respectful and culturally appropriate. We will seek advice on the understanding of research and preferences for community engagement and the process of seeking consent for research directly from Aboriginal leaders and community members.

- Local interpreters employed as Community Navigators allow research participants to speak in their language of preference, including all major local languages of the Fitzroy Valley.

- Community Navigators provide cultural guidance to visiting researchers to ensure that local cultural protocols are observed throughout the research process.

- Due to limited capacity and availability of interpreters, data analysis will be conducted in English so some subtleties of local Aboriginal language may have been lost in translation.

- This is a relatively small study in a discreet geographical setting, although likely applicable to similar remote communities elsewhere in Australia, findings may not be generalisable to all Indigenous communities.

conducted in a culturally respectful way, it can adversely affect participation rates and the quality of the research. ${ }^{3}$ Our systematic literature review of research, which describes or evaluates the process for seeking consent and the preference or understanding of the consent process for research with Indigenous communities, identified few relevant publications. ${ }^{1}$ Few detailed the methods used when seeking consent, including use of videos, flip charts or local researchers to interpret information for Indigenous participants. ${ }^{1}$ Even fewer evaluated whether this information 
was understood or presented in a way that is preferred by Indigenous participants. ${ }^{1}$ It should be noted that since there is such variety of Indigenous populations around the world, the United Nations does not have a specific definition for 'Indigenous'. ${ }^{4}$ This term will be used when referring to populations in a general sense; however, when publications specify the name of a group of people such as 'Aboriginal', that term will be used.

Guidelines for research with Indigenous communities recommend that Indigenous community members be involved in all stages of the research process. ${ }^{5}$ Strong trusting relationships between outside researchers and local community partners are essential. ${ }^{5}$ In this paper, we describe the research methods for The Picture Talk Project, a qualitative research project conducted together with remote Aboriginal communities of the Fitzroy Valley in the Kimberley region of Western Australia. In the Picture Talk Project, we will interview community leaders about the community engagement and consent process for research, conduct community focus groups about the individual consent process and publish and present findings. We hope The Picture Talk Project will support Indigenous people like those living in the Kimberley to have a more ethical, collaborative experience when participating in research and empower them to guide the research agenda to address community priorities while embedding cultural protocol into each step of the research process. ${ }^{2}$

\section{Invitation from the community}

Following the Lililwan Project on fetal alcohol spectrum disorder (FASD) prevalence, ${ }^{6-14}$ the Picture Talk Project team was invited by Aboriginal leaders of the Fitzroy Valley to explore community understanding of research and consent. ${ }^{2}$ The Lililwan project was initiated after communities leaders noticed that some of their children looked different and had trouble learning, and expressed concern that these children would not remember the dreamtime stories to be passed down to the next generation. ${ }^{12}{ }^{13}$ Because alcohol use in pregnancy was common, they wondered if these children might have FASD, so invited researchers to come to the Fitzroy Valley and investigate the issue. ${ }^{11-13}$ The project was initiated by and conducted in partnership with Aboriginal community leaders following community consultation and agreement that it was an important issue to investigate. ${ }^{11-13}$ Local Aboriginal researchers were employed as community navigators to interpret for those whose first language was not English and provide cultural guidance to the non-Aboriginal researchers. ${ }^{2}{ }^{6}$ Aboriginal and non-Aboriginal team members guided each other so that the research was both scientifically rigorous and culturally relevant. ${ }^{26}$ The Lililwan Project was so well received that in 2010 the Social Justice Commissioner for Aboriginal and Torres Strait Islander people, Mr Michael Gooda, reported that it should set an example for research with Aboriginal people. ${ }^{15}$ The Picture Talk Project was subsequently initiated, and researchers were invited to reflect with communities of the Fitzroy Valley on how and why the Lililwan
Project worked so well and to discuss the processes that should underpin research engagement and consent for the future.

\section{Why this study is important}

This research protocol uses collaborative, culturally respectful, flexible research methods to allow for participants to be in a space where they may speak freely about their experiences and opinions of research. Findings from this study will inform the approach of future studies with Indigenous communities.

\section{Study aims}

The Picture Talk Project is a community-based project conducted with local Aboriginal leaders of the Fitzroy Valley that aims to examine the community engagement and consent process. This will be achieved by interviewing Aboriginal community leaders about community consent and the research engagement process and holding focus groups with Aboriginal community members about their experiences with research and the individual consent process. Both groups will be asked how they would like research to be conducted in the future. Findings will be analysed with input from Aboriginal leaders and co-published in peer-reviewed journals and a local Fitzroy Valley community report as well as co-presented to the Fitzroy Valley communities and the wider scientific community.

\section{METHODS AND ANALYSIS}

\section{Study design}

Qualitative methods were chosen for the Picture Talk Project to enable deep exploration of participants' perspectives about research and the consent process. ${ }^{16}$ The protocol was designed in collaboration with Aboriginal leaders to ensure that local cultural protocols were embedded within each step of the research process. Results will be reported in line with the 32-item checklist for the Consolidated Criteria for Reporting Qualitative research (COREQ) guidelines. ${ }^{17}$ Semistructured interviews will be conducted with Aboriginal community leaders, and focus groups will be conducted with Aboriginal community members using 'research topic yarning' and 'collaborative yarning'. ${ }^{18} 19$ These methods are considered the best cultural match ${ }^{20}$ for gathering information with Aboriginal people. ${ }^{18} 1921$ Focus groups provide a way for participants to validate one another and avoid a potential power imbalance between participants and researchers. ${ }^{18}$

\section{Setting}

The Fitzroy Valley in northern Western Australia has Fitzroy Crossing town at its centre and 45 small communities within a $200 \mathrm{~km}$ radius. ${ }^{22}$ There are four major language groups in these communities: Walmajarri, Wangkatjungka, Gooniyandi and Bunuba as well as Kija, Nikinya language groups. ${ }^{22}$ The Fitzroy Valley is home to approximately $4500,80 \%$ of whom are Aboriginal. ${ }^{22} 23$ The whole 
of the Fitzroy Valley is classified as 'very remote' (ARIA score $>9.08-12$. ARIA, Accessibility/Remoteness Index of Australia). ${ }^{24}$ Some communities are only accessible via open (unsealed) roads that are often inaccessible during the annual wet season. ${ }^{25}$ This setting creates many challenges for both local community members and visiting researchers, with limited access to resources, transportation, emergency services and healthcare. In addition, potential participants are less engaged in research while coping with the extreme heat in the build-up to the wet season with days up to $49^{\circ} \mathrm{C}\left(120^{\circ} \mathrm{F}\right)$. Cultural protocols need to be observed such as Law (taking young members through traditional initiation) and 'Sorry Business' (mourning) which impact on the availability of local research team members and potential participants. ${ }^{25} 2627$

\section{Study timeline}

The project is being conducted between January 2012 and December 2017, and the project outline is shown in figure 1. Data collection and verification will occur from 2012 to 2017. Publication and presentation of final results will occur during 2017 and 2018.

\section{Research team}

A partnership was formed between leaders of the local Aboriginal organisations of the Fitzroy Valley community and academic researchers of organisations based in Sydney and Darwin. The chief investigators of The Picture Talk Project are JO, past Chief Executive Officer (CEO) of Marninwarntikura Women's Resource Centre (MWRG) ${ }^{28}$ and MC, CEO of Nindilingarri Cultural Health Services (NCHS) ${ }^{29}$ based in the Fitzroy Valley community; HD'A, Aboriginal researcher, the Menzies School of Research, Darwin $^{30}$; and researchers EE, AM and PhD Student and trainee in paediatrics EF from the University of Sydney who all have experience working in the remote communities of the Fitzroy Valley. ${ }^{2}$ The Picture Talk Project protocol was developed in collaboration with all partners. The team received cultural guidance and support from TL, CEO of the Kimberley Aboriginal Law and Culture Centre (KALACC), ${ }^{31}$ and Percy Bulagardie and Annette Kogolo from the Kimberley Interpreting Service, ${ }^{32}$ Fitzroy Valley. In addition to Aboriginal leaders overseeing the research process, local Aboriginal community members are employed as Community Navigators to interpret language and provide cultural guidance to EF a non-Aboriginal researcher.

\section{Researcher skills and specialised training}

According to the COREQ guidelines, it is important to declare the background, qualifications and training of the researchers involved in research with Indigenous communities. ${ }^{17}$ The Picture Talk Project constitutes EF's PhD at the University of Sydney, supervised by EE, AM, HD'A and Gaynor Macdonald. EE is a Professor of Paediatrics and Child Health, and Consultant Paediatrician who has worked in the Fitzroy Valley since 2009 and was the Chief Investigator for the Lililwan Project. AM is a psychologist and epidemiologist with Inuit family members (Canada). Both work at the University of Sydney. HD'A is an Aboriginal researcher from the Kimberley, a registered nurse, midwife and public health researcher at the Menzies Institute in Darwin. Gaynor Macdonald is an anthropologist at the University of Sydney. EF worked as a researcher on The Lililwan Project research team in 2011. She completed a Health Research Methods and Ethics course at the University of Sydney, and training for qualitative research with QSR International NVivo. ${ }^{33}$ All non-Aboriginal researchers have received cultural awareness training including from Nindilingarri Cultural Health Services ${ }^{27}$ and Aboriginal people in the Fitzroy Valley.

\section{Community Navigators}

Local Aboriginal researchers will be employed and trained as community navigators by The Picture Talk Project team. ${ }^{2}$ A Community Navigator is defined by JO and $\mathrm{MC}$ as someone who is Aboriginal, who is local to the community, respected by the community, can interpret language and provide guidance on cultural protocol, and has experience working in both Aboriginal and Western worlds. A different Community Navigator is required for each language group within Fitzroy Crossing. A male Navigator is required when speaking with men and a female Navigator is required for women. Community Navigators guide researchers in cultural protocols regarding kinship ties and skin-name relationships. For example, an Aboriginal man cannot sit in close proximity to his mother-in-law by skin-name relation. Community Navigators on the research team are connected with all four major language groups of the Fitzroy Valley (Walmajarri, Wangkatjungka, Gooniyandi and Bunuba as well as other language groups of the Kimberley such as Nikinya and Kija). This ensures adherence with cultural protocols, while simultaneously allowing community navigators to gain experience in Western research approaches. ${ }^{2}$ Community Navigators will work in partnership with EF to recruit participants; seek community and individual consent; conduct interviews and focus groups; verify transcripts and coding; present findings to the Fitzroy Valley Community and national and international conferences; and report findings through publications. ${ }^{2}$ A similar approach was taken during the Lililwan Project. ${ }^{6}$

\section{COMMUNITY ENGAGEMENT AND COMMUNITY CONSENT Relationships formed with Aboriginal communities}

Trusting, respectful relationships were formed between the local Aboriginal community and non-Aboriginal researchers over several years prior to the commencement of the study. ${ }^{2}$ During the Lililwan Project, which was overseen by JO and MC, the authors worked closely with community navigators and researchers. ${ }^{6-14} \mathrm{EF}$ and EE are biased towards the success of the Lililwan Project, which they witnessed first hand. ${ }^{17} \mathrm{EF}$ is doing this project to advocate on behalf of the Aboriginal communities for research methods that are culturally respectful and inclusive. A 


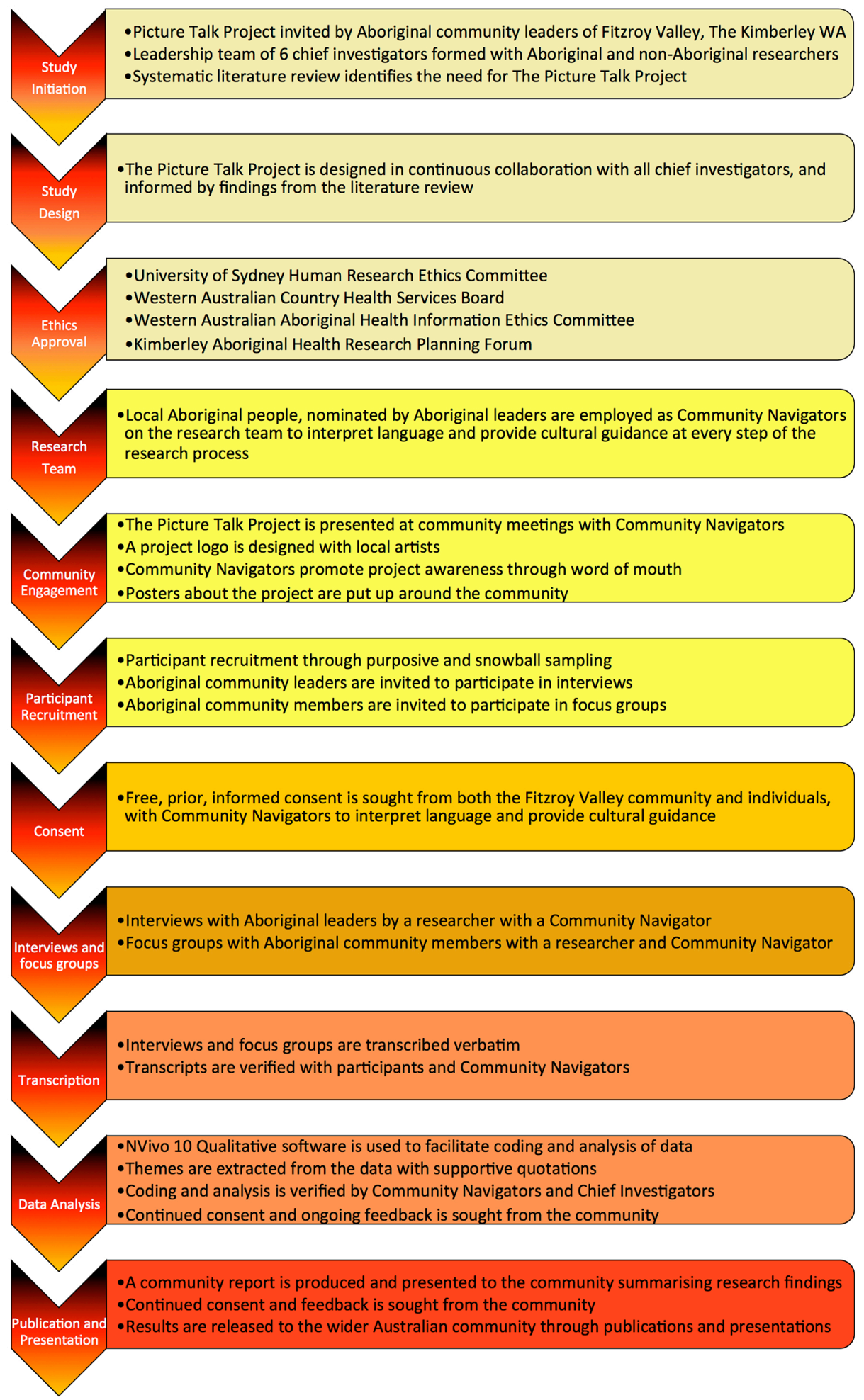

Figure 1 The Picture Talk Project Protocol.

provisional budget and flexible timeline has been developed to account for any unexpected expenses or delays. EF will make regular trips to the Fitzroy Valley to meet face to face with local Aboriginal research team members and ensure ongoing consent from the community. There will be regular meetings conducted with the key organisations of the Fitzroy Valley, namely Marninwarntikura Women's Resource Centre; Nindilingarri Cultural Health
Services; the Kimberley Aboriginal Law and Culture Centre; Karriyili Adult Education Centre; The Fitzroy Valley Men's Shed; Marra Worra Worra; Mangkatja Art Gallery and the Fitzroy Valley Future Forum. All of these organisations will be engaged and consulted about The Picture Talk Project research protocol over a number of visits. As noted by Mr Harry Yungabun, a local Aboriginal leader, health worker and community navigator for The 


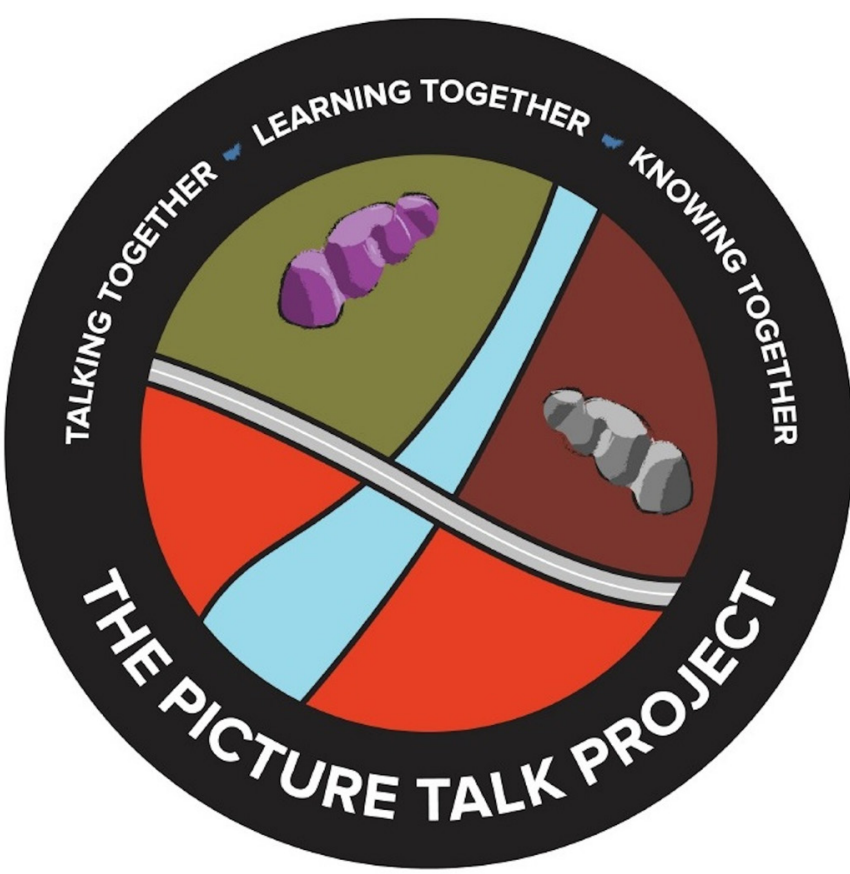

Figure 2 The Picture Talk Project Logo.

Picture Talk Project: 'Every time you come back, people open up to you more'.

\section{Project identity}

Following the Lililwan Project, Aboriginal leaders invited researchers to explore the community's understanding of research and the consent process, including the use of visual aids, for example, pictures, to explain the research. The name 'The Picture Talk Project' was given by Marmingee Hand (Aboriginal leader, school teacher and Chairperson of the Walmajarri language group of the Fitzroy Valley ${ }^{2}$ ). The name was inspired by the use of pictorial flip cards as a visual aid when seeking consent for research for the Lililwan Project, ${ }^{6}$ a method suggested by $\mathrm{MC}^{29}$ as a culturally appropriate way of seeking consent.

Community navigator Sandra Nugget and local artist Neil Carter from KALACC ${ }^{31}$ worked with EF to design a project logo (figure 2) and motto: 'Talking together, learning together, knowing together.' The logo represents all the major language groups and shows the Fitzroy River (blue), the life force of the town, intersecting with the Great Northern Highway, representing the junction between Aboriginal and Western worlds. ${ }^{2}$ The four quadrants represent the main language groups of The Valley-the purple hills to the north are the King Leopold Ranges (soon to be renamed Wilinggin Conservation Park ${ }^{34}$ ) in Bunuba country; the Black Hills of the east represent Gooniyandi country and the Great Sandy Desert in the south represents Walmajarri, Wangkatjungka, Nyikinya and Kija country. ${ }^{22}$ The black band surrounding the logo represents local Aboriginal people whom visitors are required to work with if they wish to enter local Aboriginal communities. ${ }^{2}$

This project logo will be used as a visual way of communicating what the project is about and identifying researchers. Through explaining what the logo represents, people will understand the purpose of the research. The logo will be printed on uniforms for research workers, and mugs and tote bags to be given to participants. The logo will be used on magnets for project cars and posters for car windows and elsewhere so community onlookers may read about The Picture Talk Project and approach the research team if they wish.

\section{Participants}

There will be two types of participants: Aboriginal community leaders/elders and Aboriginal community members. Aboriginal community leaders of Fitzroy Crossing are defined by JO as any person who is respected by the community, holds local cultural knowledge, has experience working with both the Aboriginal and Western worlds, and is nominated to represent and advocate for the community. Community leaders include elders whose positions are based on the cultural hierarchy of the community. It was considered acceptable to invite community leaders to be interviewed by EF in the presence of a community navigator. ${ }^{2}$ In contrast, it was deemed more culturally acceptable to hold focus groups with Aboriginal community members, rather than interviews, because many community members have had few interactions with research and Western protocols. ${ }^{18-21}$ Some focus groups will include Aboriginal community members who are parents and carers of children of the Fitzroy Valley, some of whom may have participated in the Lililwan Project. All participants will be invited to participate by a community navigator. The community navigator will be present during all interviews and focus groups to support participants.

\section{Recruitment}

In research with Aboriginal communities, both community and individual consent are required in order for the project to proceed. ${ }^{1}$ To obtain community consent, presentations will be made to key Aboriginal organisations in the Fitzroy Valley and community consent will be requested and granted from senior community members at these meetings. Individual consent will not be sought until community consent is obtained. ${ }^{1}$

Recruitment of community leaders will be through purposive sampling. ${ }^{16} 3536$ Aboriginal community leaders will be visited by community navigators who will invite them to learn more about the study and to consider participation.

In contrast, recruitment of community members will be through passive snowball sampling. ${ }^{16} 3536$ As recommended by the Aboriginal community leaders JO and MC, who are chief investigators, The Picture Talk Project will be advertised in posters; presented at community meetings and friends and family members of the community navigators; members of local Aboriginal organisations; and other research participants will be invited in person to participate. It will be up to Aboriginal community members to approach the research team or community 
navigators if they are interested in being involved in focus groups. Snowball sampling works well in such a close-knit community, as there is a very strong communication line between families. The composition of focus groups may influence the discussion so groups will by stratified by sex and age in order to encourage active participation from all parties. ${ }^{37} 38$ Male participants will be recruited into a separate focus group to females, ${ }^{37}$ the community navigator will also be of the same sex. In addition, the community navigator will only work with focus group participants with whom they have good rapport and an established connection through language group or kinship. Participants will be aged 18 years and older. Traditionally, if an Aboriginal elder is present, younger participants let their elder answer questions posed to the group. ${ }^{38}$ To encourage active participation of all members, focus groups will include participants of a similar age. ${ }^{38}$ If within a community a participant wishes to be accompanied by their elder, the research team will not exclude them from the focus group as a sign of respect. In this way, the project is designed to show respect for culturally recognised differences between the sexes and cultural protocols. ${ }^{37}$

Potential participants may nominate the time and place that is most convenient for them to be introduced to $\mathrm{EF}$ by the community navigator. $\mathrm{EF}$ and community navigators will work together to explain the scope of the research project using a participant information sheet and consent form in plain English and seek signed or verbal consent (witnessed by the community navigator) from each participant. They will then collect basic demographic information from each participant including age group, sex, education, language group, language of preference and cultural knowledge. It will be made clear that participants can decline to participate at any time and if they chose to do so the community navigator will investigate why they chose to decline.

Participants may nominate the community navigator they feel most comfortable to work with. Community members participating in focus groups may select which group of participants they would like to share a focus group with. The number of participating leaders and community members required for this study will be guided by what is scientifically rigorous yet culturally appropriate. For qualitative research, data will continue to be collected and analysed until all of the themes are 'saturated'. ${ }^{38}$ In order to be deemed culturally appropriate, it is imperative that a certain number of elders, leaders and community members are consulted from each of the main language groups included, both males and females as guided by the Aboriginal leaders and community navigators on the research team. In the Picture Talk Project, we aim to interview all the CEOs of the approximately 20 local Aboriginal owned organisations, including elders and chairpersons from all four main language groups. We aim to conduct approximately six focus groups with three to eight community members based within local organisations and living in communities in Fitzroy Crossing town, as well as a sample from very remote outlying communities. We aim to conduct at least one group where the participants are male. In addition, we aim to recruit participants to focus groups from the four main language groups and a variety of age groups.

We will record whether any focus group participants were involved with The Lililwan Project ${ }^{67}$ and its consent process because their prior experience may mean they are regarded as experts and that others might feel less likely to contribute. Exact numbers of focus groups will not be set and will be determined by what issues are discussed in each session and the saturation of themes and who is available at the time of data collection.

\section{Data collection}

A topic guide will be developed for the interviews and focus groups to ensure steps taken prior to collection of data are consistent (see online supplementary additional file 1 and supplementary additional file 2 . This will include instructions about when to start the tape recorder if consent is granted. The content of the interview and focus group questions is based on findings from the literature review. ${ }^{12}$ The research questions were informed by qualitative research methods. ${ }^{16} 18192133-42$ The language of the questions was guided by JO and MC and the community navigators. The delivery of questions will be guided by the community navigators at the time of data collection. $^{2}$

\section{Interviews with leaders}

Aboriginal community leaders will participate in semistructured interviews in the format of 'research topic yarning'. 'Yarning' is an Aboriginal way of conversing and connecting through storytelling. ${ }^{18}$ This culturally appropriate method, as described by Dawn Bessarab (an Aboriginal researcher), involves starting the conversation with what she describes as 'social yarning' where the researcher makes casual conversation with the participant in order to find common ground and potential connections through family or community and consequently gain trust and rapport. ${ }^{18}$ The researcher will then formalise the conversation by announcing the start of research questions and commence 'research topic yarning' where questions are asked in a semistructured style and any form of response is encouraged, including in a conversational or storytelling format. Aboriginal community leaders will be asked about their experiences with research and the process of community and individual consent. Interview questions (see online Supplementary additional file 3) will be asked in English by EF and interpreted by the community navigator into the language preferred by the Aboriginal leader if required. Interviews will be kept flexible and reactive to participants' responses-including both verbal and body language. If the community navigator reads that the body language starts to look closed off or disengaged, questions will be asked in a different way. Time will be allowed for silence after a question is asked. With consent, interviews will be either voice 


\title{
Excerpt from The Lililwan Project Participant Information Statement
}

\author{
Marulu (Precious), The Lililwan (little ones) Project \\ Fetal Alcohol Spectrum Disorders (FASD) Prevalence Study \\ Stage 2 - Interdisciplinary health and developmental assessments in children
}

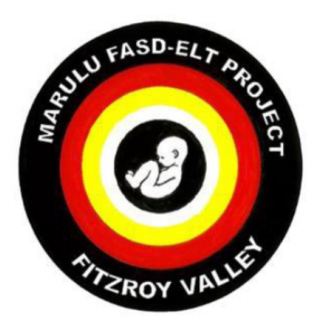

\section{What is the project about?}

The project involves health and development checks on children to see which areas they are strong in, and which areas they need more help in.

The checks we do will be similar to what the local children's doctor and Allied health team (Aboriginal health worker, speech and language pathologist, physiotherapist, school nurse, occupational therapist, eye doctor and audiologist) would normally do in the child health clinic. The checks will take around 4 hours and will be spread over 2 visits to the clinic. There are no side effects or risks expected with the checks.

If you agree to participate, your child will be seen by the eye doctor to check their vision and also have their hearing tested. With your permission, we will take a photograph of your child's face to see if they have the special face shape of children with Fetal Alcohol Syndrome. It is important to do this so that we can be sure of the diagnosis and understand the special needs of the child and get help in school or home for them if you think it is needed.

We would like to take a photograph of all the children, even if there was no alcohol used in the pregnancy. If you would like a copy of the photo we will print one out for you to keep.

On another visit your child will be checked by a children's doctor for their physical health, including listening to their heartbeat and breathing. The doctor may also ask questions about the pregnancy with this child, and the child's health and development.

The Allied health team will do some tests to see how the child is doing with memory and thinking, their behaviour at school and home, speaking, understanding of language and drawing and how coordinated and strong their muscles are. When we test the language we would like to take a short video of the child telling a story about going bush. The reason for this is so that local Aboriginal people who are experts in language can help to check the child's language including communicating by signing with their lips, eyes, facial expressions and hands.

Figure 3 Excerpt from the Lililwan Project Participant Information Statement. 


\section{Children's Doctor Check-up}

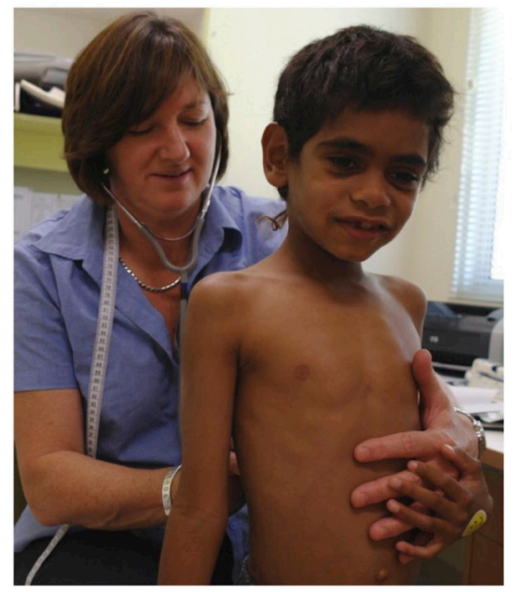

Figure 4 Excerpt from the pictorial flip chart used with forms to seek consent for the Lililwan Project.

recorded or responses hand written and transcribed later into a Microsoft Word document.

\section{Focus groups with community members}

Aboriginal community members will participate in focus groups in a format similar to 'collaborative yarning', ${ }^{18}$ which creates a space in which participants can feel comfortable and speak freely. ${ }^{19}$ The focus group will first start with 'social yarning' as described above in order to gain trust and rapport. ${ }^{18}$ The conversation will then be

\section{Children's Doctor Check-up}

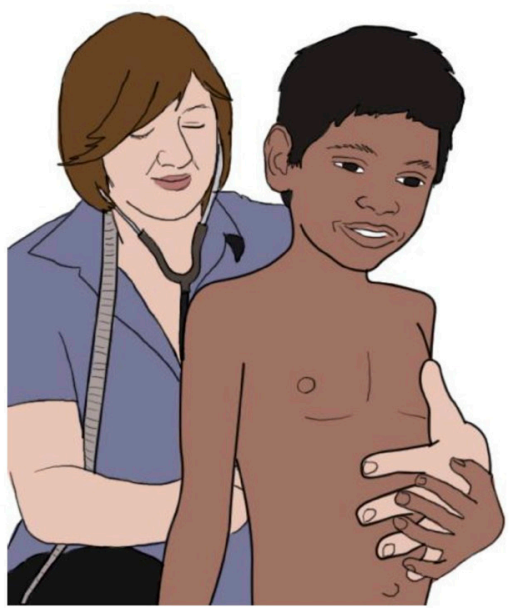

Figure 5 Cartoon version of photograph on the pictorial flip chart as example of alternative media consent. formalised to 'collaborative yarning' within the focus group, which is described as a yarn that occurs between two or more people who are discussing a research topic which may lead to new understandings. ${ }^{18}$ The advantage of the focus group format is that it creates a forum in which issues arise such as community attitudes, values and beliefs that might not have otherwise been brought to light in a one-to-one interview. ${ }^{39}{ }^{40}$ In addition, a focus group creates the capacity for the group to speak with one voice and reach consensus, which is in line with the group-focused Aboriginal way of knowing. ${ }^{21}$ Aboriginal community members will be asked about their experiences with research and the individual consent process. Focus group participants will be provided with an example of a participant information statement and (figure 3) an excerpt from the pictorial flip chart (figure 4) that was used with parents and carers in the Lililwan Project and asked to share their thoughts and preference. In addition, focus group participants will be asked to comment on a newly created cartoon version (figure 5) of the photographs shown in figure 3 as a potential medium for future consent materials. Focus group questions (see online Supplementary additional file 4) will be spoken in English and interpreted by the community navigators into the language preferred by participants. EF and the community navigators will facilitate focus groups together, encouraging the quieter participants, and managing the more dominant voices to ensure that all participants can share their opinions.

\section{Data transcription and processing}

All interviews and focus group recordings will be transcribed verbatim and handwritten responses will be typed into Microsoft Word documents. Transcripts will be verified with participants and community navigators. Data will be uploaded into NVivo10 qualitative software to facilitate the coding process. ${ }^{33}$

\section{Data analysis}

A research diary will be kept by EF to record the date, time, duration and setting of each interview and focus group, noting who is present and the first impressions of the research process. Initial codes will be created by EF immediately after each session based on the main topics discussed. ${ }^{35-38} 4344$ Additional notes will be made by EF about current community events such as 'Sorry Business' (mourning), ${ }^{27}$ which can affect participation rates and the mood of participants.

Transcripts from individual interviews and focus groups will be analysed separately by EF. A coding topic guide will be created by EF for the initial analysis of transcripts. Transcripts will be uploaded into NVivo10 qualitative software to facilitate coding. ${ }^{33}{ }^{43}$ Coding of data will be conducted by EF line by line and derived through deductive and inductive processes using grounded theory. ${ }^{41}$ When using NVivo software, one unit of analysis from the data is called a node. This node might be 'river' which is coded when participants discuss the river. A node hierarchy 
will be formed using this process and cross-referenced against all transcripts. ${ }^{3644}$ The node hierarchy is developed when nodes are collapsed into each other if they are similar and divided if there is enough data for them to be declared two entities. These top-level nodes will then be reviewed along with their linked text. Following this, overarching themes will be synthesised from this analysis and compared back against the data, known as the 'constant comparison method'. ${ }^{36}$ Rich quotes from participants will be identified to support these themes. ${ }^{33} 3843$ Coding will continue until all themes are saturated and no new information is derived. ${ }^{1635-38} 43-47$ In addition, key lessons learnt from the content within the initial interviews and focus groups will be employed when engaging with the participants for subsequent interviews or focus groups. In this way, a further layer of grounded theory ${ }^{41}$ will be applied to ensure that knowledge gained about culturally respectful research is put into practice with the continued guidance of the community navigator working alongside EF.

EF will code all transcripts to ensure coding is consistent. Community navigators who are local Aboriginal people will code random segments of data from both the interviews and focus groups, and this will be verified against coding done by EF to ensure reliability. ${ }^{44}$ Research participants as well as investigators JO and MC will be encouraged to give verbal feedback on findings individually or during community presentations and workshops. ${ }^{17}$

\section{ETHICS APPROVAL AND DISSEMINATION \\ Core values}

The Picture Talk Project follows guidelines from the Lowitja Institute, AIATSIS and NHMRC guidelines, for conduct of research with Aboriginal and Torres Strait Islander peoples in order to ensure that cultural protocols are respected and embedded into each step of our research process. ${ }^{5447-49}$ This project upholds the six core values identified as important when conducting research with Aboriginal communities: respect, equality, reciprocity, survival and protection, responsibility and above all is conducted in the right spirit and with integrity. ${ }^{47}$ This project seeks to ensure that a free, prior, informed consent is obtained from all participants and community leaders for all stages of the research.

\section{Focus group consent}

When signing consent to participate in focus groups, participants will be asked to ensure the confidentiality of discussions. Participants will be informed that they may withdraw from the session at any time but they are not able to request that their answers be erased/destroyed because this would require destroying material provided by others in the group.

\section{De-identification and storage of data}

Individual participant data will be de-identified and participants will be allocated a study code number.
Focus groups will be numbered and participants coded accordingly. Voice-recorded material will be transcribed. Quotations used in research reports to capture the rich qualitative data obtained during interviews and focus groups will be de-identified. Data will be securely stored electronically on a password protected network drive and only nominated study investigators will have access to the data.

Throughout the study, hard copy data will be stored securely in a locked filing cabinet in the University of Sydney-Sydney Medical School Discipline of Child and Adolescent Health, Sydney Children's Hospital Network (Westmead) or Marninwarntikura Women's Resource Centre, Fitzroy Valley. At the conclusion of the study, all data will reside at the University of Sydney and de-identified data will be accessible to the research community on application.

As per guidelines set out by the University of Sydney Human Research Ethics Committee, all data including audio files will be retained for a period of 7 years.

\section{Consent for dissemination}

Community and individual consent will be sought for publication, dissemination and presentation of all aspects of this research to date.

\section{DISCUSSION}

Key strengths of this study are that it was prioritised and initiated by the community, will be conducted in partnership with Aboriginal community leaders who are Chief Investigators of this study and that two thirds of the research team are local Aboriginal people. The Picture Talk Project is designed to be culturally sensitive while maintaining scientific rigour through analysis using grounded theory. This qualitative study will explore how researchers can form collaborative partnerships with Aboriginal communities to ensure that informed consent and respect for Aboriginal culture are embedded at every stage of the process. The Picture Talk Project seeks to embody these values and lead by example. Local interpreters are trained and employed as community navigators on the research team to provide guidance in language and cultural protocol throughout the research processfrom consent seeking to presentation of results. It must be noted that the Picture Talk project is an exemplar of a research framework working with communities. Cultural protocols may vary from one community to another, and it is important to be flexible and respond to needs identified by the community navigators. ${ }^{5}$ For example, there may be 'Sorry Business' (mourning for a death in the family) about which the community navigator but not the researcher is aware. A particular family or community might have a special ritual associated with mourning for their loss that takes a set amount of time and this might delay research. A limitation of this study is that some nuances of language may be lost through the process of interpreting between local languages and English. 
This is this first study with a specific focus on community engagement and the process of seeking consent for research in Aboriginal communities. This research will provide a voice for remote-dwelling Aboriginal people of the Fitzroy Valley, and provide unique insights into understanding of research and preferences about how it should be conducted to embody respect for Aboriginal culture and values. Aboriginal communities are not against research itself but want research that is conducted in the right way. Following the successful Lililwan Project, the Picture Talk Project was proposed by Aboriginal community members and is a 'community-driven' partnership with external researchers.

\section{Author affiliations}

${ }^{1}$ Discipline of Child and Adolescent Health, University of Sydney Medical School, Sydney, Australia

${ }^{2}$ Discipline of Child and Adolescent Health, Sydney Children's Hospitals Network Randwick and Westmead, Sydney, Australia

${ }^{3}$ Nindilingarri Cultural Health Services, Fitzroy Crossing, Australia

${ }^{4}$ Marninwarntikura Women's Resource Centre, Fitzroy Crossing, Australia

${ }^{5}$ Nulungu Research Institute, The University of Notre Dame, Broome, Australia

${ }^{6}$ Kimberley Aboriginal Law and Culture Centre, Fitzroy Crossing, Australia

${ }^{7}$ School of Public Health, University of Sydney Medical School, Sydney, Australia

${ }^{8}$ Dalla Lana School of Public Health, University of Toronto - Mississauga,

Mississauga, Canada

${ }^{9}$ Epidemiology Division, The George Institute for Global Health, Sydney, Australia ${ }^{10}$ Division of Education and Capacity Building, Menzies School of Health Research, Darwin, Australia

Acknowledgements We thank Ms Sandra Nuggett, Ms Joy Nuggett, Mr Percy Bulagardie, Mr HarryYungabun, Ms Marilyn Oscar, Ms June Smith for their excellent work as Community Navigators on the research team—providing language andcultural guidance. Thank you to Ms Annette Kogolo for introducing Community Navigators and continuing to validate and verifying transcript coding. Thankyou to Ms Sharon Eadie for support with ethics applications and transcriptions and Mrs Anastasia Kindrakewich for assisting with transcriptions. We would like to thank Ms Sandra Nuggett and Mr Neil Carterfor their work into the design of the project logo and Mr John Watts, Ms Erin Fitzpatrick and Ms Katherine Fitzpatrick for their assistance with computergraphics. Thank you to Ms Marmingee Hand who created the name of this project. Finally, thank you to the Aboriginal communities of the Fitzroy Valley for inviting, collaborating and participating in this research project.

Contributors EF led the study and the writing of the paper. EE initiated and provided oversight to the study and input in its planning, conduct and reporting. J0 and MC invited this study, advised on the protocol design and are the chief cultural advisors as Aboriginal community leaders of the Fitzroy Valley, The Kimberley and had input into the manuscript. TL provided cultural guidance and support from the men's side at the Kimberley Aboriginal Law and Culture Centre and had input into the manuscript. AM and HA contributed to the design of the study and the writing of the paper. All authors reviewed and approved the final manuscript.

Funding EF is supported by an AVANT Doctors in Training Part-Time scholarship; the Australian Federation of Graduate Women Barbara Hale Fellowship; and the Rowan Nicks Russell Drysdale Fellowship. EE is supported by a National Health and Medical Research Council Fellowship (no 1021480). AM was supported by a University of Sydney Fellowship (2012-2015) and an NHMRC Translating Practice into Research (TRIP) Fellowship (2016-2018) (no 1112387).

Competing interests None declared.

Patient consent Obtained.

Provenance and peer review Not commissioned; externally peer reviewed.

Open Access This is an Open Access article distributed in accordance with the Creative Commons Attribution Non Commercial (CC BY-NC 4.0) license, which permits others to distribute, remix, adapt, build upon this work non-commercially, and license their derivative works on different terms, provided the original work is properly cited and the use is non-commercial. See: http://creativecommons.org/ licenses/by-nc/4.0/ (c) Article author(s) (or their employer(s) unless otherwise stated in the text of the article) 2017. All rights reserved. No commercial use is permitted unless otherwise expressly granted.

\section{REFERENCES}

1. Fitzpatrick EF, Martiniuk AL, D'Antoine $\mathrm{H}$, et al. Seeking consent for research with indigenous communities: a systematic review. BMC Med Ethics 2016;17:65.

2. Fitzpatrick EFM, Macdonald G, Martiniuk ALC, et al. The picture talk project: starting a conversation with community leaders on research with remote aboriginal communities of Australia. BMC Med Ethics 2017:18:34.

3. Dodson M, Williamson R. Indigenous peoples and the morality of the human genome diversity project. J Med Ethics 1999;25:204-8.

4. United Nations. State of the World's Indigenous Peoples. United Nations Division for Social Policy and Development of the Department of Economic and Social Affairs, 2015.

5. Laycock A, Walker D, Harrison N, et al. Researching Aboriginal Health: a practical guide for researchers. Melbourne, Australia: The Lowitja Institute: Australia's National Institute for Aboriginal and Torres Strait Islander Research, 2011.

6. Fitzpatrick JP, Elliott EJ, Latimer J, et al. The Lililwan Project: study protocol for a population-based active case ascertainment study of the prevalence of Fetal Alcohol Spectrum Disorders (FASD) in remote Australian aboriginal communities. BMJ Open 2012;2:e000968.

7. Fitzpatrick JP, Latimer J, Carter M, et al. Prevalence of fetal alcohol syndrome in a population-based sample of children living in remote Australia: the Lililwan Project. J Paediatr Child Health 2015;51:450-7.

8. Fitzpatrick JP, Latimer J, Ferreira ML, et al. Prevalence and patterns of alcohol use in pregnancy in remote Western Australian communities: The Lililwan Project. Drug Alcohol Rev 2015;34:329-39.

9. Fitzpatrick JP, Latimer J, Olson HC, et al. Prevalence and profile of neurodevelopment and Fetal Alcohol Spectrum Disorder (FASD) amongst Australian aboriginal children living in remote communities. Res Dev Disabil 2017;65:114-26.

10. Elliott E, Latimer J, Fitzpatrick J, et al. There's hope in the valley. J Pa ediatr Child Health 2012;48:190-2.

11. Latimer J Elliott E FJea. Marulu: The Lililwan Project Fetal Alcohol Spectrum Disorders (FASD) prevalence study in the fitzroy valley: a community consultation. The George Institute for Global Health 2010.

12. Hogan M. Yajilarra. A documentary. Fitzroy Crossing, Australia, 2008.

13. Hogan M. A documentary for the Lililwan Project: Marulu. Fitzroy Crossing, Australia, 2009.

14. Tsang TW, Lucas BR, Carmichael Olson $\mathrm{H}$, et al. Prenatal Alcoho Exposure, FASD, and child behavior: a meta-analysis. Pediatrics 2016;137:e20152542.

15. Gooda M. Chapter 3: From community crisis to community control in the Fitzroy Valley - Social Justice Report 2010. Canberra, Australia: Australian Human Rights Commission, Parliament House 2010.

16. Rice PL, Ezzy D. Qualitative research methods. Melbourne, Victoria: Oxford University Press, 1999.

17. Tong A, Sainsbury P, Craig J. Consolidated criteria for reporting qualitative research (COREQ): a 32-item checklist for interviews and focus groups. Int J Qual Health Care 2007;19:349-57.

18. Bessarab $\mathrm{D}, \mathrm{Ng}$ 'andu $\mathrm{B}$. Yarning about yarning as a legitimate method in indigenous research. Int Jour of Crit Ind Stud 2010;3:37-50.

19. Geia LK, Hayes B, Usher K. Yarning/Aboriginal storytelling: towards an understanding of an Indigenous perspective and its implications for research practice. Contemp Nurse 2013;46:13-17.

20. Manley AJ B, Cornell S. What is cultural match and why is it so important? Native Nations Institute for Leadership, Management, and Policy(University of Arizona) andHarvard Project on American Indian Economic Development, 2008. http://fngovernance. org/resources_docs/Why_Is_Cultural_Match_So_Important_ Presentation.pdf

21. Willis $E$, Pearce $M$, Jenkin T. Adapting Focus group methods to fit aboriginal community-based research. Qual Res 2005:5:112-23.

22. Morphy F. Population, people and place: the fitzroy valley population project. Centre for Aboriginal Economic Policy Research: Australian National University, 2010. Working paper 70.

23. ABS. Australian Bureau of Statistics Census QuickStats: Fitzroy Crossing, 2011.

24. AlHW. LFaRH UIn: , ed. Rural, regional and remote health: a guide to remoteness classifications. Canberra, Australia, 2004.

25. DHAC. Meauring Remoteness Accessibility/Remoteness Index of Australia (ARIA). 2001. 
26. AIATSIS. Guidelines for Ethical Research in Indigenous studies Australia. Australian Institute of Aboriginal and Torress Strait Islander Studies, 2012.

27. Wynne-Jones MHA, Byers D, Stanley D, et al. Aboriginal grief and Ioss: a review of the literature. 2016;16:1-9.

28. MWRC. Marninwarntikura women's resource centre Fitzroy Crossing, Australia. https://www.mwrc.com.au/

29. NCHS. Nindilngarri cultural health services Fitzroy Crossing, Australia. http://www.nindilingarri.org.au/

30. MSHR. Menzies school of health research, Darwin, Australia. http:// www.menzies.edu.au/

31. KALACC. Kimberley aboriginal law and culture centre Fitzroy Crossing, Australia. http://www.kalacc.org.au/

32. KIS. Kimberley interpreting service. http://www.kimberleyinterpreting. org.au/

33. QSR. NVivo 10 qualitative software. http://www.qsrinternational.com/

34. Government of Western Australia. New national park for the North Kimberley. Australia, 2017.

35. Sobo EJ. Culture and meaning in health services research: a practical field guide. Walnut Creek, California: Left Coast Press, 2009.

36. Crabtree BMW. Doing qualitative research: research methods of primary care. Newbury Park, CA: Sage Publications, 1992.

37. Cuneen C. Criminology, criminal justice and Indigenous people: a dysfunctional relationship. Current Issues Crim 2008;20:323.

38. Green J, Thorogood N. Qualitative methods for health research. London: SAGE, 2004.
39. Christopher S, Watts V, McCormick AK, et al. Building and maintaining trust in a community-based participatory research partnership. Am J Public Health 2008;98:1398-406.

40. Coreil J. Group interview methods in community health research. Med Anthropol 1995;16:193-210.

41. Fereday J, Muir-Cochrane E. Demonstrating rigor using thematic analysis: a hybrid approach of inductive and deductive coding and theme development. Int J Qual Methods 2006;5:80-92.

42. Liamputtong P, Ezzy D. Qualitative research methods. 2nd edn. Oxford, UK: Oxford University Press, 2005.

43. Bazeley P. Qualitative Data Analysis with Nvivo. London: Sage, 2007.

44. Bradley EH, Curry LA, Devers KJ. Qualitative data analysis for health services research: developing taxonomy, themes, and theory. Health Serv Res 2007;42:1758-72.

45. Strauss A, Corbin J. The basics of qualitative research: grounded theory procedures and techniques. Newbury Park, CA: Sage Publishing, 1990.

46. Hannes K, Lockwood C. Synthesizing qualitative research: choosing the right approach. London, United Kingdom: Wiley-Blackwell, 2012.

47. NHMRC. NHMRCIn: , ed. Values and Ethics: guidelines for conduct of aboriginal and Torres Strait Islander health research. Australia, 2003.

48. NHMRC. NHMRCKeeping Research on Track: a guide for aboriginal and Torres Strait Islander people about Health Research and Ethics. In: , ed. Australia, 2005.

49. NHMRC. NHMRCRoad Map II: a strategic framework for improving aboriginal and Torres Strait Islander health through research. In: , ed. Australia, 2010 